\title{
AN ANTI-DIFFUSION BASED EULERIAN INTERFACE-SHARPENING ALGORITHM FOR COMPRESSIBLE TWO-PHASE FLOW WITH CAVITATION
}

\author{
Keh-Ming Shyue \\ Department of Mathematics \\ National Taiwan University \\ Taipei, Taiwan 10617 \\ Email: shyue@ntu.edu.tw
}

\section{SUMMARY}

We describe a novel interface-sharpening approach for the efficient numerical simulation of compressible two-phase flow with cavitation. The algorithm uses a five-equation transport model developed by Allaire et al. (J. Comput. Phys. 181 (2002) 577-616) as the basis, and incorporates both the auxiliary antidiffusive terms to the equations as a model for interface sharpening and an energy-preserving pressure cutoff procedure as a model for cavitation. A standard fractional-step method is employed to solve the proposed model equations in two separate steps, yielding an easy implementation of the algorithm. Sample numerical results for underwater explosions in two dimensions are shown to demonstrate the feasibility of the algorithm for practical problems.

\section{INTRODUCTION}

Consider an unsteady, inviscid, compressible homogeneous two-phase flow that is modeled by a five-equation transport equations of the form

$$
\begin{aligned}
\partial_{t}\left(\alpha_{1} \rho_{1}\right)+\nabla \cdot\left(\alpha_{1} \rho_{1} \vec{u}\right) & =0, \\
\partial_{t}\left(\alpha_{2} \rho_{2}\right)+\nabla \cdot\left(\alpha_{2} \rho_{2} \vec{u}\right) & =0, \\
\partial_{t}(\rho \vec{u})+\nabla \cdot(\rho \vec{u} \otimes \vec{u})+\nabla p & =0, \\
\partial_{t} E+\nabla \cdot(E \vec{u}+p \vec{u}) & =0, \\
\partial_{t} \alpha_{1}+\vec{u} \cdot \nabla \alpha_{1} & =0,
\end{aligned}
$$

for the principal motion of the state variables such as the partial densities, momentum, total energy, and volume fraction, respectively (cf. [1]). Here $\rho_{k}$ and $\alpha_{k} \in[0,1]$ denote in turn the $k$ th phasic density and volume fraction for $k=1,2, \rho=\alpha_{1} \rho_{1}+\alpha_{2} \rho_{2}$ denotes the total density, $\vec{u}$ the vector of particle velocity, and $p$ the mixture pressure. For simplicity, we assume that the constitutive law for the material of interest can be characterized by the stiffened gas equation of state

$$
p_{k}=\left(\gamma_{k}-1\right) \rho_{k} e_{k}-\gamma_{k} p_{\infty, k},
$$

where $e_{k}$ is the specific internal energy, $\gamma_{k}>1$ is the ratio of specific heats, and $p_{\infty, k}$ is a pressure-like constant. As usual, $E=\rho e+\rho|\vec{u}|^{2} / 2$ is the total energy with $\rho e=\sum_{k=1}^{2} \alpha_{k} \rho_{k} e_{k}$ representing the internal energy and $|\vec{u}|$ being the Euclidean distance of the velocity vector. In this model, we have the saturation condition $\alpha_{1}+\alpha_{2}=1$ imposed for the volume fractions as well.

If we assume further the isobaric closure where $p_{1}=p_{2}=p$ in a region that contains more than one fluid component, we may derive easily the expression for the mixture pressure

$$
p=\left(\rho e-\sum_{k=1}^{2} \alpha_{k} \frac{\gamma_{k} p_{\infty, k}}{\gamma_{k}-1}\right) / \sum_{k=1}^{2} \frac{\alpha_{k}}{\gamma_{k}-1},
$$

and also the expression for the mixture acoustic impedance

$$
\rho c^{2}=\sum_{k=1}^{2} \alpha_{k} \frac{\gamma_{k}\left(p+p_{\infty, k}\right)}{\gamma_{k}-1} / \sum_{k=1}^{2} \frac{\alpha_{k}}{\gamma_{k}-1},
$$

where $c$ is the mixture speed of sound. Then it can be shown that this five-equation model is hyperbolic when each physically relevant value of the state variables of the flow are defined in the region of thermodynamic stability, see [1] for the detail.

It is clear that since the volume fraction plays an important role in this compressible two-phase flow modelling, the devise of a robust scheme that attains non-oscillatory, positivity-preserving resolution of volume fraction near the interface before and after wave interactions is highly desirable and necessary when solving this model system numerically. In this work, motivated by the recent success of the anti-diffusion Eulerian interface sharpening approach proposed by So, Hu, and Adams [2] for viscous incompressible two-phase flow, we are interested in a novel extension of the method from incompressible to compressible two-phase flow with and without cavitation. Our goal here is to describe the basic idea of this extension, and give a preliminary assessment of the method for some sample problems, see [3-5] for previous works in this direction. 
It should be mentioned that for incompressible flow interface sharpening of some kind (cf. [6-13]) is a popular technique that is applied in an underlying advection scheme to compute sharp solution profile for the volume fraction equation arising from the modelling of numerical mixing between two different incompressible fluid components in a control volume. Since the resulting volume fraction solution is used solely to the definition of the averaged material quantities that are present as coefficients in the Navier-Stokes equations, it is a common practice to decouple this volume fraction computation from the remaining part of the flow solver, yielding an efficient implementation of the method.

For compressible flow, however, because of the intrinsic coupling of genuinely nonlinear and linearly degenerate waves in the solution states, the development of an efficient interface sharpening method requires some thoughts, see [14] for an example based on artificial interface compression.

\section{ANTI-DIFFUSION INTERFACE SHARPENING MODEL}

To begin with, we recall that the anti-diffusion interface sharpening model proposed by So, $\mathrm{Hu}$, and Adams [2] for solving the volume-fraction transport equation

$$
\partial_{t} \alpha_{1}+\vec{u} \cdot \nabla \alpha_{1}=0
$$

with given $\vec{u}$ and discontinuous initial $\alpha_{1}$ takes the form

$$
\partial_{t} \alpha_{1}+\vec{u} \cdot \nabla \alpha_{1}=-\frac{1}{\mu} \nabla \cdot\left(\varepsilon \nabla \alpha_{1}\right),
$$

where $\varepsilon>0$ is the diffusion coefficient, and $\mu$ is a free parameter. To approximate (5) numerically, a fractional step method that consists of the following steps in each time iteration is employed:

\section{Algorithm 1}

(1) (Advection step) Solve the homogeneous part of the model equation, i.e., (4), using a monotone scheme [15] over a time step $\Delta t$.

(2) (Anti-diffusion step) Take the solution obtained in step 1 as the initial condition, and solve the model equation with only source term

$$
\partial_{\tau} \alpha_{1}=-\nabla \cdot\left(\varepsilon \nabla \alpha_{1}\right)
$$

using a simple explicit method, for example, over a time step $\Delta \tau$ towards a "sharp layer".

Here $\tau=t / \mu$ is a scaled time variable. Note that numerical regularization is required such as employing the MINMOD limiter to stabilize the computation of $\nabla \alpha$ and so the flux $\varepsilon \nabla \alpha$ in step 2 of the method (cf. [16, 17]). Sample numerical results in two dimensions are present that show the feasibility of this interfacesharpening approach for passive advection problems and problems arising from incompressible two-phase flow [14].

To extend this method for interfaces governed by the fiveequation compressible two-phase model (1), as before (cf. [18]), it is useful to begin with an interface-only problem where both the pressure and each component of the particle velocities are constant in the domain, while the other variables such as the density and the material quantities are having jumps across some interfaces. Then we write (1) in the following non-conservative form,

$$
\begin{aligned}
& \partial_{t}\left(\alpha_{1} \rho_{1}\right)+\vec{u} \cdot \nabla\left(\alpha_{1} \rho_{1}\right)+\alpha_{1} \rho_{1} \nabla \cdot \vec{u}=0, \\
& \partial_{t}\left(\alpha_{2} \rho_{2}\right)+\vec{u} \cdot \nabla\left(\alpha_{2} \rho_{2}\right)+\alpha_{2} \rho_{2} \nabla \cdot \vec{u}=0, \\
& \partial_{t}(\rho \vec{u})+\vec{u} \nabla \cdot(\rho \vec{u})+\rho \vec{u} \cdot \nabla \vec{u}+\nabla p=0, \\
& {\left[\partial_{t}\left(\rho \frac{1}{2}|\vec{u}|^{2}\right)+\vec{u} \nabla \cdot\left(\rho \frac{1}{2}|\vec{u}|^{2}\right)+\rho \frac{1}{2}|\vec{u}|^{2} \nabla \cdot \vec{u}\right]+} \\
& \quad\left[\partial_{t}(\rho e)+\vec{u} \cdot \nabla(\rho e)+\rho e \nabla \cdot \vec{u}\right]+\nabla \cdot(p \vec{u})=0, \\
& \partial_{t} \alpha_{1}+\vec{u} \cdot \nabla \alpha_{1}=0,
\end{aligned}
$$

yielding easily the basic transport equations for this interfaceonly problem

$$
\begin{aligned}
\partial_{t}\left(\alpha_{1} \rho_{1}\right)+\vec{u} \cdot \nabla\left(\alpha_{1} \rho_{1}\right) & =0, \\
\partial_{t}\left(\alpha_{2} \rho_{2}\right)+\vec{u} \cdot \nabla\left(\alpha_{2} \rho_{2}\right) & =0, \\
\vec{u}\left(\partial_{t} \rho+\vec{u} \cdot \nabla \rho\right) & =0, \\
\frac{|\vec{u}|^{2}}{2}\left(\partial_{t} \rho+\vec{u} \cdot \nabla \rho\right)+\left[\partial_{t}(\rho e)+\vec{u} \cdot \nabla(\rho e)\right] & =0, \\
\partial_{t} \alpha_{1}+\vec{u} \cdot \nabla \alpha_{1} & =0 .
\end{aligned}
$$

Having (5) in mind for the volume fraction, it should be sensible to assume that the anti-diffusion model for the phasic densities $\alpha_{1} \rho_{1}$ and $\alpha_{2} \rho_{2}$ take the form

$$
\partial_{t}\left(\alpha_{1} \rho_{1}\right)+\vec{u} \cdot \nabla\left(\alpha_{1} \rho_{1}\right)=-\frac{1}{\mu} \nabla \cdot\left(\varepsilon \nabla \alpha_{1} \rho_{1}\right)
$$

and

$$
\partial_{t}\left(\alpha_{2} \rho_{2}\right)+\vec{u} \cdot \nabla\left(\alpha_{2} \rho_{2}\right)=-\frac{1}{\mu} \nabla \cdot\left(\varepsilon \nabla \alpha_{2} \rho_{2}\right)
$$

respectively. By summing up the above phasic-density equations, we have our model for the total density

$$
\partial_{t} \rho+\vec{u} \cdot \nabla \rho=-\frac{1}{\mu} \nabla \cdot(\varepsilon \nabla \rho) .
$$

With that, to ensure the velocity remains at a constant state across the interfaces the momentum equation should be modified by

$$
\vec{u}\left(\partial_{t} \rho+\vec{u} \cdot \nabla \rho\right)=-\frac{1}{\mu} \vec{u} \nabla \cdot(\varepsilon \nabla \rho) .
$$

Furthermore, to ensure the pressure retain in equilibrium, using (2), (5), and (8), it is not difficult to show that the energy 
equation should be modified also by

$$
\begin{gathered}
\frac{|\vec{u}|^{2}}{2}\left(\partial_{t} \rho+\vec{u} \cdot \nabla \rho\right)+\left[\partial_{t}(\rho e)+\vec{u} \cdot \nabla(\rho e)\right]= \\
-\frac{1}{\mu}\left[\frac{|\vec{u}|^{2}}{2} \nabla \cdot(\varepsilon \nabla \rho)-\Delta(\rho e) \nabla \cdot\left(\varepsilon \nabla \alpha_{1}\right)\right],
\end{gathered}
$$

where $\Delta(\rho e)=\rho_{2} e_{2}-\rho_{1} e_{1}$.

Since in general we are interested in shock wave problems as well, we should apply the anti-diffusion terms described above only locally near the interfaces. For this reason, it is common to introduce an interface indicator, denoted by $H_{I}$, to the model so that it takes effect near the interfaces only, and has no effect on the other genuinely nonlinear shock and rarefaction waves.

In summary, with the stiffened gas equation of state (2), the anti-diffusion interface-sharpening model we propose to solve compressible two-phase flow takes the form

$$
\begin{aligned}
\partial_{t}\left(\alpha_{1} \rho_{1}\right)+\nabla \cdot\left(\alpha_{1} \rho_{1} \vec{u}\right) & =-\frac{1}{\mu} H_{I} \mathscr{D}_{\alpha_{1} \rho_{1}}, \\
\partial_{t}\left(\alpha_{2} \rho_{2}\right)+\nabla \cdot\left(\alpha_{2} \rho_{2} \vec{u}\right) & =-\frac{1}{\mu} H_{I} \mathscr{D}_{\alpha_{2} \rho_{2}}, \\
\partial_{t}(\rho \vec{u})+\nabla \cdot(\rho \vec{u} \otimes \vec{u})+\nabla p & =-\frac{1}{\mu} H_{I} \mathscr{D}_{\rho \vec{u}}, \\
\partial_{t} E+\nabla \cdot(E \vec{u}+p \vec{u}) & =-\frac{1}{\mu} H_{I} \mathscr{D}_{E}, \\
\partial_{t} \alpha_{1}+\vec{u} \cdot \nabla \alpha_{1} & =-\frac{1}{\mu} \mathscr{D}_{\alpha_{1}} .
\end{aligned}
$$

Here, for convenience, we use the notation $\mathscr{D}_{z}=\nabla \cdot(\varepsilon \nabla z)$ to denote the differential term for the state variable $z$ for $z=\alpha_{1}$, $\alpha_{1} \rho_{1}, \alpha_{2} \rho_{2}, \rho$ appearing in (5), (6), (7), and (8), respectively. Thus, without causing any confusion, we may use the notation $\mathscr{D}_{\rho \vec{u}}=\vec{u} \mathscr{D}_{\rho}$ to denote the differential term appearing in (9) and $\mathscr{D}_{E}=\left(|\vec{u}|^{2} / 2\right) \mathscr{D}_{\rho}+\Delta(\rho e) \mathscr{D}_{\alpha_{1}}$ for the differential term appearing in (10).

To find approximate solutions of our interface sharpening model (11), we use a similar fractional-step method as described in AlgORITHM 1 where in step 1 a standard wave propagation method developed by LeVeque $[19,20]$ is employed to solve the homogeneous part of the model equations, and in step 2 the model equation with the source term only is solved using a standard explicit finite difference method (cf. [21]) for the anti-diffusion heat equation towards a "sharp layer" (in practice, only 1 or 2 iterations are enough for the interface-sharpening purpose).

Here the local interface indicator $H_{I}$ is considered to be a Heaviside function in the volume-fraction jump whose value is one when this jump in the nearby state exceeds some tolerance and zero otherwise. Alternatively, we may choose $H_{I}$ to be a hyper-tangent function of the volume fraction, see [14], or use the other measure based on physical quantities such as entropy, pressure, and velocity on this matter. Note that we have taken the diffusion coefficient $\varepsilon$ to be a function of the local velocity that varies both in space and time in the computation shown below, which is unlike the case considered in [14] where only a single diffusion constant is used in the entire domain at each time.

\section{CAVITATION MODEL}

Before proceeding further, we pause to discuss a simple pressure cutoff procedure that can be included in (11) as a model for cavitation, see [22-25] and references therein for other stateof-the-art cavitation models. In this case, when the pressure $p$ computed by (2) is lower than a cavitation threshold such as the saturated vapor pressure $p_{\text {sat }}$, we assume it to be the value of this quantity, and so the cutoff phasic internal energy may be set by $\left(\rho_{k} e_{k}\right)_{\text {sat }}=\left(p_{\text {sat }}+\gamma_{k} p_{\infty, k}\right) /\left(\gamma_{k}-1\right)$ in a cavitated region for $k=1,2$. With that, it is quite common to implement this cavitation model as a post-processing step in a numerical method, where after each time step, we apply the usual non-conservative energy correction

$$
E:=E_{\text {Sat }}=\sum_{k=1}^{2} \alpha_{k}\left(\rho_{k} e_{k}\right)_{\text {Sat }}+\frac{1}{2} \rho|\vec{u}|^{2}
$$

see [26], or alternatively the energy-preserving volume-fraction correction proposed here,

$$
\alpha_{1}:=\left(\alpha_{1}\right)_{\mathrm{sat}}=\frac{\rho e-\left(\rho_{2} e_{2}\right)_{\mathrm{sat}}}{\left(\rho_{1} e_{1}\right)_{\mathrm{sat}}-\left(\rho_{2} e_{2}\right)_{\mathrm{sat}}} .
$$

Here $\left(\alpha_{1}\right)_{\text {sat }}$ can be derived directly from the $\alpha$-weighted average of $\rho e$. Note that, in the former case, the other state variables such as $\alpha_{1} \rho_{1}, \alpha_{2} \rho_{2}, \rho \vec{u}$, and $\alpha_{1}$ are kept as constants during the correction, while in the latter case, all the conservative variables in (1) are kept as constants.

It should be noted that no matter what post-processing correction, i.e., (12) or (13), is applied to the algorithm, the speed of sound in the cavitation region is computed consistently with (3). In addition, it is not difficult to see that the difference in the total energy between these two different corrections is equal to a constant times the difference in the volume fraction, i.e.,

$$
\Delta E=\left[\left(\rho_{2} e_{2}\right)_{\mathrm{sat}}-\left(\rho_{1} e_{1}\right)_{\mathrm{sat}}\right] \Delta \alpha_{1},
$$

where $\Delta z=z-z_{\text {sat }}$ for $z=E$ and $\alpha_{1}$. Thus, even though there may be no significant compression or expansion of the volume fraction during some stages of the cavitation inception and termination where both $\Delta \alpha_{1}$ and $\Delta E$ are small, the energy-preserving approach should be a more viable one to be used in this cutoff model as opposed to the non-conservative energy approach.

\section{NUMERICAL EXAMPLES}

We now present sample results for underwater explosions in two dimensions obtained using our interface sharpening method with cavitation. Additional results that further validate the proposed method will be reported elsewhere.

\section{UNDERWATER EXPLOSIONS NEAR A FLAT WALL}

As a first example, we consider a model underwater explosion problem studied by Pishevar and Amirifar [27] near a solid 
wall. In this test, the initial condition consists of a stationary circular explosive charge of radius $1 \mathrm{~m}$ that is placed at the origin of a rectangular region $\left(x_{1}, x_{2}\right) \in[-15,15] \times[-3,15] \mathrm{m}^{2}$ that is full of water. Here, for simplicity, the explosive is modeled as a perfect gas with the state variables $\rho=1,270 \mathrm{~kg} / \mathrm{m}^{3}, p=9,000 \mathrm{bar}$, $\gamma=2.0$, and $p_{\infty}=0$, and the water is modeled as a stiffened gas with $\rho=1,000 \mathrm{~kg} / \mathrm{m}^{3}, p=1$ bar, $\gamma=4.4$, and $p_{\infty}=6,000$ bar. In carrying out the computation, the saturation pressure we use in the cutoff model for cavitation is $p_{\text {sat }}=0.3619$ bar, and the boundary conditions are solid wall on the bottom, and nonreflecting on the remaining sides of the domain.

Due to the presure difference, the explosion of the gas bubble leads to an outward-going shock wave in water, an inward-going rarefaction wave in gas, and a contact discontinuity lying in between that separates the gas and water. As times go along, the outward-going shock wave would be reflected from the flat wall that generates shock and bubble interaction, creating a reflected rarefaction wave that lower the pressure and the incept of cavitation in a region between the bubble and the wall.

Figure 1 shows a sequence of pressure contours at four different times $t=1,3,4,6 \mathrm{~ms}$ obtained using our anti-diffusion interface sharpening algorithm using a $300 \times 180$ grid, where in the graph we have also included contour lines indicating the locations of the gas bubble as well as the region of cavitation. From the figure, we observe the general features of the solutions as described above. It is easy to see that there is no spurious oscillations in the pressure near the bubble interface, showing that our anti-diffusion algorithm works in a satisfactory manner for this problem.

The time histories of the pressure and the velocity at the center of the wall are shown in Fig. 2, where a fine grid solution obtained using the method without the anti-diffusion is included for comparison. From the figure, we observe, on the one thing, good agreement between the results with and without anti-diffusion, and on the other thing, minor effect to the pressure and velocity field due to sharpening of interface at this plotted location at the least. We note that, qualitatively our results are in good agreement with the one appeared in [27] using an adaptive Arbitrary Lagrangian-Eulerian method and a barotropic model for water with cavitation, see [28,29] for similar results but are obtained using different constitutive laws and numerical methods.

\section{UNDERWATER EXPLOSION IN A RIGID CYLINDER}

Our second example is concerned with an explosion of a spherical gas bubble in a cylinder that contains water. Similar to the setup considered in $[22,28,29]$, we take a cylinder that is of $0.0889 \mathrm{~m}$ in diameter and $0.2286 \mathrm{~m}$ height. Initially, a stationary spherical explosive charge of diameter $0.03 \mathrm{~m}$ is placed at the center of the cylinder. Here, the explosive is modeled as a perfect gas again with the state variables $\rho=1,770 \mathrm{~kg} / \mathrm{m}^{3}, p=20,000$ bar, $\gamma=2.0, p_{\infty}=0$, and the water is modeled as a stiffened gas with the same parameters as used in the previous example. The saturation pressure we use in this test for the cavitation cutoff is $p_{\text {sat }}=0.05$ bar. Since the problem is symmetric with respect to the cylindrical axis, we only take the right-half of the cylinder during the computations. The boundary conditions we used are solid wall on the left and right, and non-reflecting on the top and

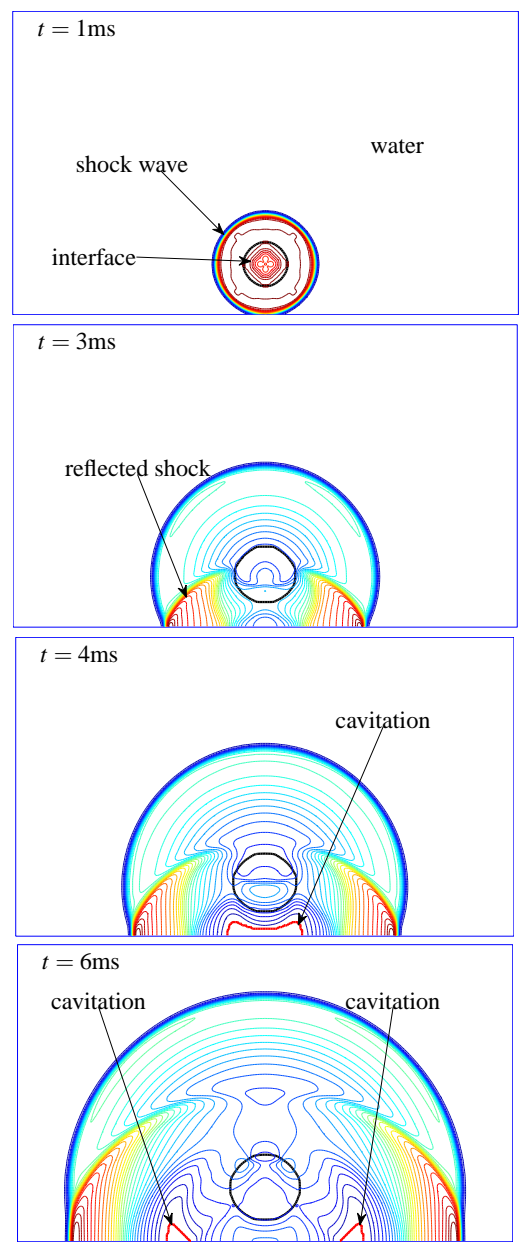

Figure 1. ANTI-DIFFUSION RESULTS FOR AN UNDERWATER EXPLOSION PROBLEM NEAR A FLAT SOLID WALL. A SEQUENCE OF PRESSURE CONTOURS IS SHOWN AT FOUR DIFFERENT TIMES 1, 3, 4, 6MS USING A $300 \times 180$ GRID.
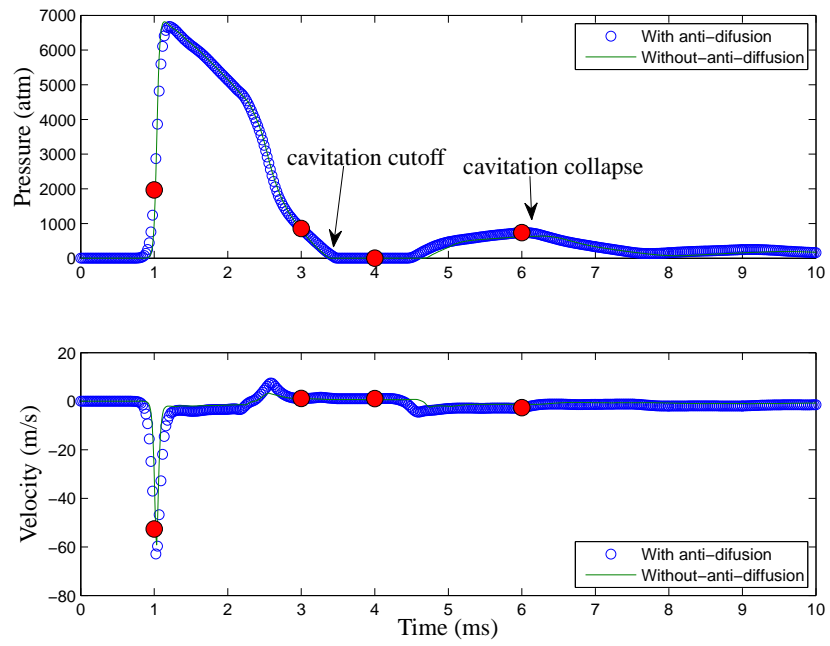

Figure 2. THE TIME HISTORIES OF THE PRESSURE AND VELOCITY AT THE CENTER OF THE SOLID WALL. THE FILLED CIRCLES IN THE GRAPH ARE THE RESULTS AT THE SELECTED TIMES SHOWN IN FIG. 1. RESULTS OBTAINED USING THE METHOD WITHOUT ANTI-DIFFUSION USING A FINER $600 \times 360$ ARE INCLUDED ALSO FOR COMPARISON. 
bottom sides.

For this problem, at the early time, the basic mechanism for the creation of the cavitation region is the same as in the previous example. That is, due to the shock wave reflection from the cylinder wall and the resulting shock-bubble interactions, a lower pressure region is formed between the bubble and the cylinder wall. However, due to the geometric effect, complicated wave interaction occurs at a later time, yielding the collapse of the original cavitation, and the formation of the others in various parts of the cylinder.

In Fig. 3, we plot a sequence of the pressure contours at four different times $t=30,45,60,120 \mu$ s obtained using our antidiffusion interface sharpening algorithm using a $140 \times 720$ grid, where we have made the graph for the solution in the whole cylindrical region so that the basic features of the solution mentioned above can be seen more clearly. From the figure, we again observe smooth behavior of the pressure field near the bubble interface computed by the algorithm.

The time histories of the pressure and the velocity at the center of the cylindrical wall are shown in Fig. 4, where a fine grid solution obtained using the method without the anti-diffusion is included for comparison. From the figure, we again observe good agreement between the results with and without anti-diffusion, and not so much effect to the pressure and velocity field due to sharpening of interface. Furthermore, we notice some qualitative agreement of solutions when our results are in comparison with those one appeared in the literature, see [22, 28, 29].

Note that to solve this problem numerically, geometric source terms have been included in the model (cf. [30]), and have been handled as one of the fractional steps before the antidiffusion step of the original algorithm.

\section{CONCLUSIONS}

We have presented a simple interface-sharpening approach based on the anti-diffusion viewpoint for the numerical resolution of compressible two-phase flow with cavitation. Numerical results for underwater explosions show sensible behavior of the anti-diffusion solutions when comparisons are made with the finer grid solutions without anti-diffusion. Ongoing work is to extend this approach further to problems with phase transitions (cf. [31,32]).

\section{ACKNOWLEDGMENTS}

The author was supported in part by the National Science Council of Taiwan Grants \#96-2115-M-002-008-MY3 and 992115-M-002-005-MY2.

\section{REFERENCES}

[1] Allaire, G., Clerc, S., and Kokh, S., 2002. "A five-equation model for the simulation of interface between compressible fluids". J. Comput. Phys., 181, pp. 577-616.

[2] So, K. K., Hu, X. Y., and Adams, N. A., 2011. "Antidiffusion method for interface steepening in two-phase incompressible flow". J. Comput. Phys., 230, pp. 5155-5177.
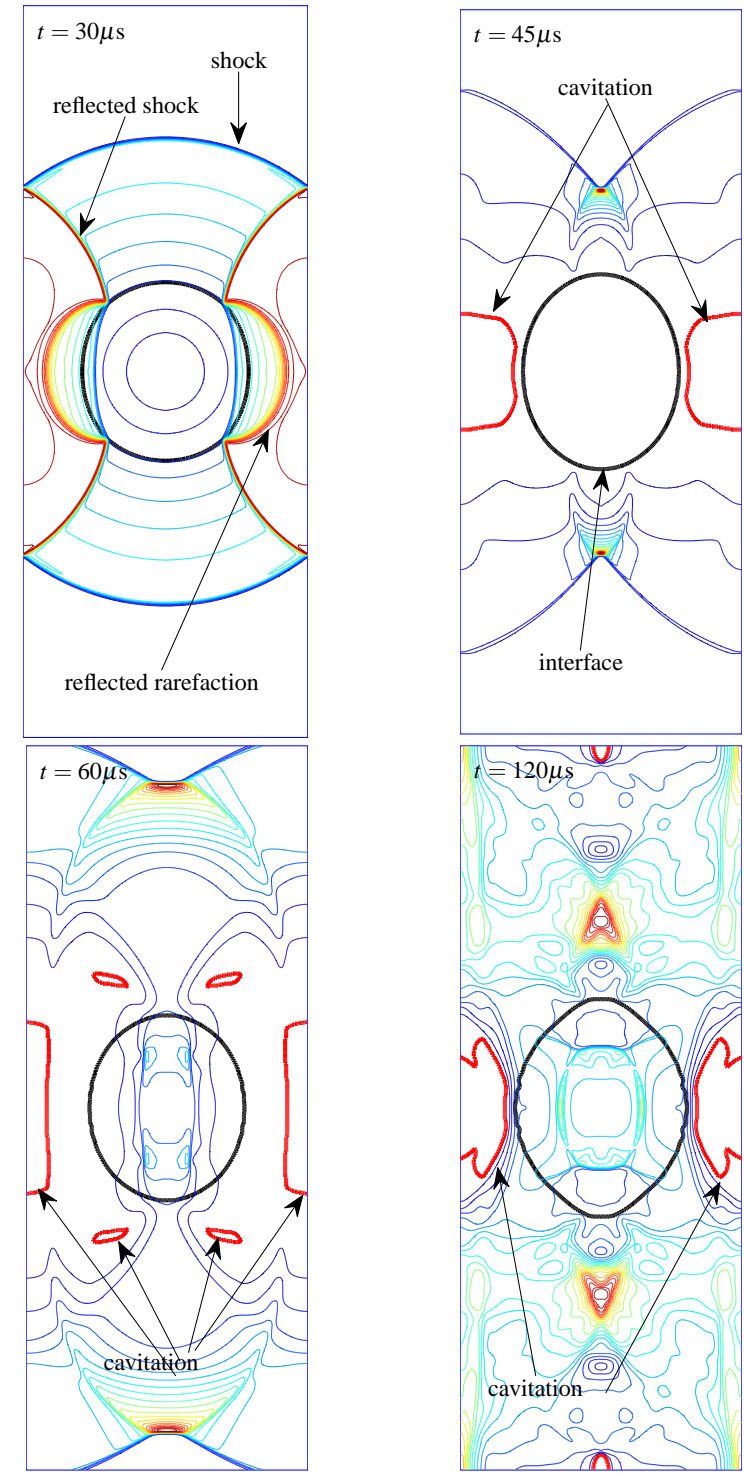

Figure 3. ANTI-DIFFUSION RESULTS FOR AN UNDERWATER EXPLOSION PROBLEM IN THE SINGLE RIGID CYLINDER. A SEQUENCE OF PRESSURE CONTOURS IS SHOWN AT FOUR DIFFERENT TIMES $t=30,45,60$, AND $120 \mu$ S USING A $140 \times 720$ GRID.

[3] Després, B., and Lagoutier̀e, F., 2001. "Contact discontinuity capturing schemes for linear advection and compressible gas dynamics". J. Sci. Comput., 16(4), pp. 479-524.

[4] Kokh, S., and Lagoutière, F., 2010. "An anti-diffusive numerical scheme for the simulation of interfaces between compressible fluids by means of a five-equation model". $J$. Comput. Phys., 229, pp. 2773-2809.

[5] Xu, Z., and Shu, C.-W., 2005. "Anti-diffusive flux corrections for high order finite difference WENO schemes". $J$. Comput. Phys., 205, pp. 458-485.

[6] Boris, J. P., and Book, D. L., 1973. "Flux-corrected transport I. SHASTA, a fluid transport algorithm that works". $J$. Comput. Phys., 11, pp. 38-69.

[7] Noh, W. F., and Woodward, P., 1976. "SLIC (simple line 

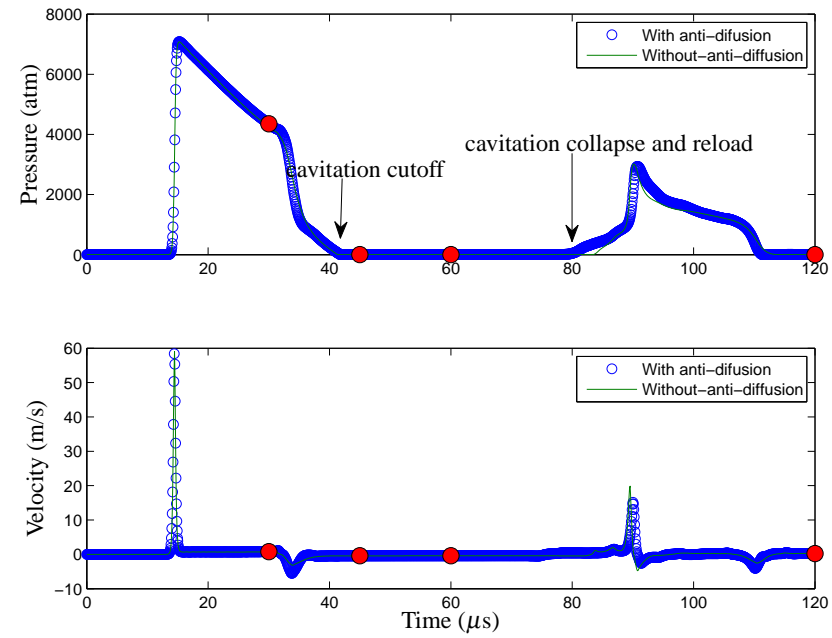

Figure 4. THE TIME HISTORIES OF THE PRESSURE AND VELOCITY AT THE CENTER OF THE CYLINRICAL WALL. THE FILLED CIRCLES IN THE GRAPH ARE THE RESULTS AT THE SELECTED TIMES SHOWN IN FIG. 3. RESULTS OBTAINED USING THE METHOD WITHOUT ANTI-DIFFUSION USING A FINER $280 \times 1440$ GRID ARE INCLUDED ALSO.

interface calculation)". In Proc. 5th Intl. Conf. on Numer. Meth. in Fluid Dynamics, A. I. van de Vooren and P. J. Zandbergen, eds., Springer-Verlag.

[8] Ubbink, O., and Issa, R. I., 1999. "A method for capturing sharp fluid interfaces on arbitrary meshes". J. Comput. Phys., 153, pp. 26-50.

[9] LeVeque, R. J., 2002. Finite Volume Methods for Hyperbolic Problems. Cambridge University Press.

[10] Xiao, F., Honma, Y., and Kono, T., 2005. "A simple algebraic interface capturing scheme using hyperbolic tangent function". Int. J. Numer. Mech. Fluids, 48, pp. 1023-1040.

[11] Xiao, F., Li, S., and Chen, C., 2011. "Revisit to the THINC scheme: A simple algebraic VOF algorithm". J. Comput. Phys., 230, pp. 7086-7092.

[12] Yokoi, K., 2007. "Efficient implementation of THINC scheme: A simple and practical smoothed VOF algorithm". J. Comput. Phys., 226, pp. 1985-2002.

[13] Youngs, D. L., 1982. “Time-dependent multi-material flow with large fluid distortion". In Numerical Methods for Fluid Dynamics, K. W. Morton and M. J. Baines, eds., Academic Press, pp. 273-285.

[14] Shukla, R. K., Pantano, C., and Freund, J. B., 2010. “An interface capturing method for the simulation of multi-phase compressible flows". J. Comput. Phys., 229, pp. 74117439.

[15] LeVeque, R. J., 1992. Numerical Methods for Conservation Laws, 2 ed. Birkhäuser-Verlag.

[16] Breuß, M., Brox, T., Sonar, T., and Weicker, J., 2005. "Stabilized nonlinear inverse diffusion for approximating hyperbolic PDEs". Proceedings scale space and PDE methods in computer vision, 3459, pp. 536-547.

[17] Breuß, M., and Welk, M., 2007. "Staircasing in semidiscrete stabilized inverse linear diffusion algorithms". J. Comput. Appl. Math., 206, pp. 520-533.
[18] Shyue, K.-M., 1998. "An efficient shock-capturing algorithm for compressible multicomponent problems". J. Comput. Phys., 142, pp. 208-242.

[19] LeVeque, R. J., 1988. "High resolution finite volume methods on arbitrary grids via wave propagation". J. Comput. Phys., 78, pp. 36-63.

[20] LeVeque, R. J., 1997. "Wave propagation algorithms for multi-dimensional hyperbolic systems". J. Comput. Phys., 131, pp. 327-353.

[21] LeVeque, R. J., 2007. Finite Difference Methods for Ordinary and Partial Differential Equations: Steady-State and Time-Dependent Problems. SIAM, Philadelphia.

[22] Liu, T. G., Khoo, B. C., and Xie, W. F., 2004. "Isentropic one-fluid modelling of unsteady cavitating flow". J. Comput. Phys., 201, pp. 80-108.

[23] Saurel, R., and Abgrall, R., 1999. "A multiphase Godunov method for compressible multifluid and multiphase flows". J. Comput. Phys., 150, pp. 425-467.

[24] Saurel, R., and LeMetayer, O., 2001. "A multiphase model for compressible flows with interfaces, shocks, detonation waves, and cavitation”. J. Fluid Mech., 431, pp. 239-271.

[25] Saurel, R., Petitpas, F., and Berry, R. A., 2009. "Simple and efficient relaxation methods for interfaces separating compressible fluids, cavitating flows and shocks in multiphase mixtures". J. Comput. Phys., 228, pp. 1678-1712.

[26] Deiterding, R., Cirak, F., and Mauch, S. P., 2009. "Efficient fluid structure interaction simulation of viscoplastic and fracturing thin-shells subjected to underwater shock loading". In Intl. Workshop on Fluid Structure Interaction, S. Hartmann, A. Meister, M. Schäfer, and S. Turek, eds., University Press GmbH, pp. 65-80.

[27] Pishevar, A. R., and Amirifar, R., 2010. "An adaptive ALE method for underwater explosion simulations including cavitation". Shock Waves, 20, pp. 425-439.

[28] Park, J., August, 2008. "A coupled runge kutta discontinuous galerkin-direct ghost fluid (rkdg-dgf) method to near-field early-time underwater explosion (undex) simulations". $\mathrm{PhD}$ thesis, Virginia Polytechnic Institute and State University. Available at http://www.openthesis.org/documents/Runge-KuttaDiscontinuous-Galerkin-Direct-591985.html.

[29] Wardlaw, Jr., A. B., and Luton, J. A., 2000. "Fluid-structure mechanisms for close-in explosions". Shock and Vibration, 7, pp. 265-275.

[30] Shyue, K.-M., 2010. "A high-resolution mapped grid algorithm for compressible multiphase flow problems". J. Comput. Phys., 229, pp. 8780-8801.

[31] Saurel, R., Petitpas, F., and Abgrall, R., 2008. "Modelling phase transition in metastable liquids: application to cavitating and flashing flows". J. Fluid. Mech., 607, pp. 313-350.

[32] Zein, A., Hantke, M., and Warnecke, G., 2010. "Modeling phase transition for compressible two-phase flows applied to metastable liquids". J. Comput. Phys., 229, pp. 29642998. 